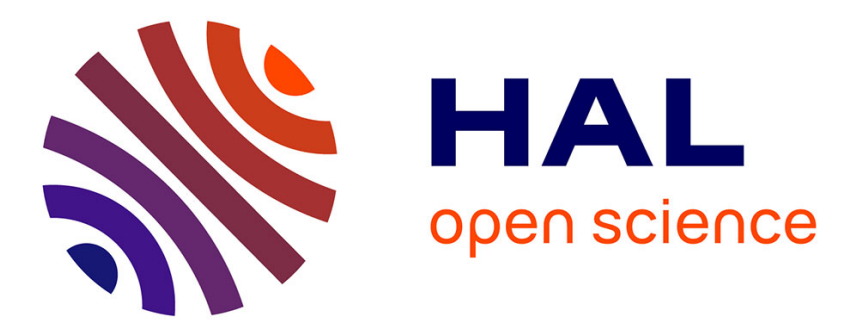

\title{
Les relations professionnelles face aux nouveaux rapports de subordination \\ Jean Saglio
}

\section{To cite this version:}

Jean Saglio. Les relations professionnelles face aux nouveaux rapports de subordination. Héloïse Petit, Nadine Thévenot. Les nouvelles frontières du travail subordonné Approche pluridisciplinaire, La Découverte, pp.235-243, 2006. halshs-00188815

\section{HAL Id: halshs-00188815 https://shs.hal.science/halshs-00188815}

Submitted on 19 Nov 2007

HAL is a multi-disciplinary open access archive for the deposit and dissemination of scientific research documents, whether they are published or not. The documents may come from teaching and research institutions in France or abroad, or from public or private research centers.
L'archive ouverte pluridisciplinaire HAL, est destinée au dépôt et à la diffusion de documents scientifiques de niveau recherche, publiés ou non, émanant des établissements d'enseignement et de recherche français ou étrangers, des laboratoires publics ou privés. 


\section{“ Les relations professionnelles face aux nouveaux rapports de subordination"}

\section{Discussion par Jean Saglio ${ }^{1}$}

Il existe une longue tradition dranalyse des situations de travail subordonnées qui consiste à dénoncer les contraintes que la domination des puissants impose aux faibles. Le ressort de lévolution se trouve alors dans la logique du capital et la connaissance des situations de travail passe par lélucidation des conditions d'optimisation de la mise en œuvre du capital. Les analyses institutionnalistes dans lesquelles s'enracinent les traditions de relations professionnelles utilisent de tout autres principes danalyse. On peut leur appliquer la remarque que faisait Jean-Maurice Verdier à propos du droit du travail : il se distingue radicalement des autres droits en ce quil nobéit pas à une logique, mais relève de l,histoire. La remarque vaut très certainement aussi plus largement pour les systèmes de relations professionnelles. Le souci de cohérence formelle est plus un obstacle qu'une aide à la compréhension de tels ensembles. Dans une telle tradition, la discussion scientifique nest donc pas la confrontation simple entre deux modèles, pas plus qu'elle n'est la recherche de la cohérence interne. Plutôt elle procède par notations partielles et ajouts limités. La présente contribution consiste donc en quelques réflexions, dont le caractère disparate n'est pas fortuit.

\section{La règle est le produit de la négociation et non de la domination seule}

Un bref rappel des apports des pères fondateurs de cette tradition analytique permet de mieux situer le point de vue adopté ici. La première référence renvoie aux travaux des Webbs sur les effets de laction syndicale, et donc à une tradition anglaise de la fin du XIXe grenoble.fr

CRISTO - CNRS, Université Pierre Mendès-France; Jeran.Saglio@upmf- 
siècle ${ }^{2}$. Crest la négociation collective qui occupe chez eux la place habituellement dévolue à la décision dans les analyses économiques. On retient ici de leur thèse lassertion que la compréhension des mécanismes de formation des règles, et donc de la négociation collective, est plus importante que la connaissance de létat du marché pour comprendre les pratiques des relations demploi et de travail. Pour connaître le niveau du salaire et ses évolutions, on va donc focaliser lattention sur lélaboration de la convention collective plutôt que de s'intéresser aux désirs du patron ou à létat du marché. Les analyses de relations professionnelles vont donc se tenir à distance des thèses libérales qui attribuent aux marchés et à eux seuls les dynamiques de transformation de lespace économique tout autant que de celles qui, à l,inverse, mettent l,accent sur la domination d'un acteur sur les autres.

La seconde référence canonique qui sous-tend l,analyse est empruntée à John Commons. Le travail salarié est à la fois, inséparablement et inéluctablement, conflit et coopération. La coopération seule, telle que rêvée dans bien des modèles microéconomiques, est une utopie. Aucune organisation de travail n'est une institution où la coopération règne seule. Réciproquement, le conflit seul conduit à la catastrophe, et l’organisation n'est pas durable. Pour autant le conflit n'est pas une dysfonction de l,organisation. Pour parvenir à prendre en compte cette difficulté, Commons incite à centrer l,analyse sur la transaction ${ }^{3}$ qui est à la fois l,échange et la règle de l'échange. On ne peut dissocier léchange de la règle de léchange, notamment dans léchange de marché. Autrement dit, les lois abstraites comme " la loi du marché " n’ont guère de sens. Il faut constamment se demander : quelle est la règle effective à ce moment sur ce marché ? Et c'est en comprenant la règle et sa formation que l’on comprend la situation. Ce qui n'est pas tout à fait le tandem coercition / consentement de Gramsci

2 Une bonne présentation synthétique de ces approts dans Morel C. 1975, Les universitaires et le mouvement ouvrier aux USA. Le cas de l'Université du Wisconsin, Thèse, Fondation nationale des Sciences Politiques, Paris.

3 Cf. l,introduction de Commons J.R. 1934, Institutional Economics, its place in political Economy, The University of Wisconsin Press, 1959, 2 Vol. ( $1^{\circ}$ éd. Macmillan, 1934) 
rappelé par Thomas Coutrot. Dans la mesure où celui-ci se réfère centralement à la relation d,autorité ; alors que conflits et coopérations sont également envisageables dans des organisations non hiérarchiques.

\section{Des dynamiques conflictuelles plurielles}

De tels présupposés amènent à discuter l,hypothèse utilisée par Thomas Coutrot selon laquelle lexplication principale réside dans la dynamique dominante que l,un des acteurs impose, et que le système est unifié autour de cette dynamique dominante. A linverse, je pose l,hypothèse que, pour comprendre ce qui se passe en France notamment l,histoire récente - il est important de prendre aussi en compte ce qui s'est joué dans cet autre lieu que sont les relations professionnelles dans les fonctions publiques. Ainsi par exemple l,importance du modèle de statut dans les débats sur les contrats de travail en France se comprend mieux quand on prend en compte les fonctions publiques et leur histoire qu'en se référant aux seules exigences du capital et de la réalisation du capital.

De même, si l’on s'intéresse aujourd,hui aux conflits du travail, on ne peut se passer de prendre en compte les spécificités des fonctions publiques. Où se passent les conflits aujourd,hui ? Où sont les forces syndicales ? Poser ces questions amène à se demander si le noyau dur du salariat aujourd'hui n'est pas constitué autour des salariés de l,État ${ }^{4}$. Crest là que sont les conflits et les syndiqués. Prendre en compte cette spécificité permet de mieux comprendre les orientations et les actions des syndicats. Et donc, une analyse focalisée exclusivement sur la dynamique du capital est insuffisante. Simultanément, accorder de lattention à cette intensité de la conflictualité dans les situations de fonction publique produit drautres questions : à lévidence la divergence des taux de conflits ne peut être reportée ni à la répression féroce qui sévirait dans le privé et empêcherait toute expression des mécontentements, ni, à l,inverse, à la détérioration relative des

4 C'est notamment la thèse de Bernard Friot, 1998, Puissances du salariat, Emploi et protection sociale à la françaíse, La Dispute. 
conditions d'emploi dans le public.

\section{Quels enjeux des luttes?}

Quels sont les enjeux des conflits? Quelles sont les revendications des subordonnés quand ils contestent la subordination? S'agit-il uniquement et toujours de contester la répartition des richesses produites? Autrement dit, la contestation de la régulation par les marchés n'est-elle qu'une contestation des résultats de la distribution ? Le détour par larticle où Edward $\mathrm{P}$. Thompson traite des émeutes populaires de la période préindustrielle ${ }^{5}$ est heuristique en la matière. II défend la thèse que dans l,Angleterre d,avant l,industrialisation et la victoire intellectuelle de Smith, des luttes tout à fait populaires et récurrentes ont pour objectif de contrer les modes de régulation par le marché que cherchent à imposer justement les bourgeois libéraux.

Ce n'est ni le coût du grain, ni les niveaux de rémunération des travailleurs populaires que contestent ces mouvements, c'est le mode de commercialisation. Ce que réclament les foules dans ces "mobs", c'est la priorité au marché local : il exigent que l’on revienne aux anciennes coutumes où l’on doit d/abord servir le marché local. Ensuite les producteurs pourront exporter. La procédure de vente, sur ce marché local est réglée: avant le second coup de cloche, il faut servir les indigents. Et enfin, la coutume fixe les outils de mesure utilisés pour les transactions. Les revendications ne portent pas sur les prix, ni sur les salaires. L'émergence ultérieure du capitalisme industriel déplace les conflits, les acteurs et les rapports de forces. C'est alors que les conflits deviennent des grèves modernes et non plus des " riots ", ils portent sur les niveaux de rémunération et non sur les formes déchanges sur les marchés, ils mobilisent les producteurs et non les consommateurs.

Peut-on transposer le raisonnement à la situation présente? Si effectivement la régulation par le marché devient dominante, ce ne seront plus les mêmes forces qui vont être porteuses. Sur ce second point je rejoins partiellement les remarques de Thomas Coutrot: les

5 Thompson E.P. 1971, "The Moral Economy of the English Crowd in the Eighteenth Century ", Past and Present, $\mathrm{n}^{\circ}$ 50, feb 1971, 76-136. 
forces contestatrices ne sont plus des "grands glacis " d'antan, et il est envisageable que les conflits futurs associent plus fortement les consommateurs.

Les enjeux ne seront plus les mêmes non plus. Le plus souvent encore, les analystes posent la question des résultats en termes de distribution et non en termes de procédures. Ce qui correspond à un moment historique particulier : les foules anglaises nous dit Thompson, ne revendiquent pas dabord en termes de quantité ou de prix des grains, mais sur les procédures d'allocation des ressources. Ce n'est pas parce que le marché libre favoriserait les uns ou défavoriserait les autres qu'il est critiqué, c'est la non visibilité des transactions qui est mise en cause. Ce n'est pas l,injustice de la redistribution qui est critiquée, c'est laccès du public au contrôle qui est revendiqué.

\section{La fin des Trente Glorieuses : quelles ruptures ?}

Pour introduire la discussion sur les rapports de forces et leurs configurations, je minspirerai des travaux de Gerald Friedman'. L'ouvrage mérite discussion, notamment quant aux méthodes de traitement des données sur les conflits du travail. Je reprendrai ici seulement l,une des pistes de compréhension de la rupture qui s'est faite dans les années 1970 en France. Friedman avance que ce qui caractérise la différence entre les deux pays au tournant du $\mathrm{XIX}^{\circ}$ et $\mathrm{XX}^{\circ}$ siècles, est un positionnement des acteurs sur léchiquier politique. Dans la situation américaine, le patronat campe au centre de léchiquier politique parce quil est, d'une certaine façon, le porte-drapeau du contractualisme. Et le contractualisme est au fondement de la pensée de la pensée républicaine américaine. Alors que dans la situation française, le patronat est considéré traditionnellement comme antirépublicain. L'alliance républicaine, et on retrouve encore cette configuration dans la Résistance et la Guerre d'Algérie, inclut les syndicats et exclut le patronat.

6 Friedman G. 1998, State making and Labor movement, France and the United Sates, 1876 - 1914, Cornell University Press 
Or après 1968 et le passage de François Ceyrac au CNPF, on peut penser que le patronat n'est plus considéré comme antirépublicain, il n'est plus une menace contre la République. Jusques et y compris au putsch des généraux dravril 1961, la République menacée venait demander le soutien des syndicats. Aujourd’hui, la République ne se sent plus menacée, ou plutôt, si elle se sent menacée, le patronat ne fait plus partie de cette menace. Cette configuration politique n'est-elle pas l,une des clefs du changement des rapports de forces à partir de la première moitié des années soixante dix.

\section{Employeur et subordination}

Dans sa contribution, Jacques Freyssinet souligne que la question de lemployeur, ainsi que celle des organisations d'employeurs, sont des clefs importantes pour comprendre létablissement des modes de régulation du travail. Même en lrabsence du regretté Jean Bunel, je me vois mal critiquer une telle assertion. Si on accepte l,idée que lemployeur est un acteur, et non seulement un agent neutre du capital qui serait strictement défini par une de ses fonctions, il faut effectivement mettre la question de lanalyse de ses stratégies à lagenda des recherches sur la subordination et ses nouvelles formes.

On peut aller au delà de ce premier souhait. Et commencer par tirer le constat que cette question est récurrente : voilà plusieurs décennies au moins que nous pouvons constater que cette question de lemployeur et des organisations d'employeurs reste le trou noir de nos approches. Le plus souvent parce qu'on suppose que leur comportement serait simple à comprendre, dicté par les contraintes des marchés et des formes de rattachement au capital. Une telle assertion est au fondement de bien des institutions, et pas seulement des discours de syndicats de salariés. On la retrouve quotidiennement dans la plupart des analyses économiques usuelles quelles soient ou non sophistiquées par l'usage d’appareil statistiques.

Comment donc éviter cette assimilation de lemployeur et du capitaliste qui constitue le piège habituel dans lequel disparaît le plus 
souvent la question des employeurs? II me semble que partir de lemployeur public - c'est-à-dire justement de celui dont le lien avec le capital n'est pas évident - est alors une méthode heuristique. Que veut dire subordination dans les situations de fonctions publiques? Les différences que l’on va observer alors relèvent-elles de la structure des organisations, des traditions professionnelles spécifiques ou des stratégies patronales ? Comment le mode de subordination s'articule-t-il sur les résistances et les conflits ? Mieux comprendre ces situations de fonctions publiques me paraît aujourd,hui l,un des moyens d,avancer dans la compréhension des autres formes de subordination.

\section{Emploi ou travail}

Jacques Freyssinet propose de renforcer le rôle des régulations de métier. C'est reconnaître que, en matière de relations professionnelles, lattention s'est focalisée jusqu'à maintenant sur des questions de conditions de travail, et que l,on cherche à s'orienter sur les conditions d'emploi. Discuter une telle proposition mérite qu'on la replace dans son contexte historique. On peut partir de la distinction que François Gaudu pointe pour le début du XX ${ }^{\circ}$ siècle entre emploi et travail'. Jusqu'en 1936, la plupart des conventions collectives sont des conventions collectives de travail; on parle également à lépoque de conventions collectives ouvrières. Elles concernent quasi exclusivement le monde ouvrier et ce qu'elles régulent, c'est le travail. À côté, on observe des systèmes notamment dans la fonction publique détat, mais aussi dans le privé, qui régulent lemploi. Mais ce sont alors plutôt des statuts que des objets de négociation.

On a bien, dans l,histoire des négociations collectives de salariés, au moins une convention qui porte directement sur lemploi. C'est la fameuse Convention d,Halluin signée juste avant la Première Guerre mondiale par laquelle les syndicats (c'est-à-dire la CGT) obtiennent, dans le bâtiment, un contrôle syndical sur lembauche. Donc on a bien une classique régulation de profession. Les syndicats contrôlent

7 Gaudu F. 1986, L'emploi dans l'entrepríse privée, Essai de théorie juridíque, Thèse, Université Paris I. 
lembauche, moyennant quoi le patron obtient la flexibilité de l'emploi. Pendant un temps, la Cour de Cassation accepte cette politique (arrêt du 24 octobre 1916); puis connaît un revirement et l,interdit.

Pourquoi ce revirement au début des années $1920^{8}$ ? Pour respecter le pluralisme syndical plaide Bonnecase. Si la liberté dradhésion au syndicat de son choix est une liberté constitutionnelle, on ne peut pas avoir un monopole syndical de lembauche. L'argumentation est d'une grande mauvaise foi chez Bonnecase, qui cherche drabord à contrer la CGT, mais l,argument doit être examiné. Aller vers un modèle de profession, est-ce aller vers le contrôle syndical de lembauche? Cela suppose une transformation notable des syndicats. Qu'on peut penser en cours notamment avec les accords de juillet 2001. C'est un changement considérable assimilable à un changement de régulation.

L'affaire est peut-être moins radicale. On peut entendre la proposition comme une suggestion de se rapprocher de lautre modèle français, où des organisations représentant les salariés ont un certain contrôle sur lembauche. C'est le cas de certain " corps" de la fonction publique. Ce sont des "syndicats" au sens de la théorie des relations professionnelles, bien quils ne soient pas rattachés à une confédération syndicale. On peut prendre lexemple des officiers de la Marine Nationale : ce sont d'autres militaires qui sont déjà en poste et qui organisent et contrôlent le recrutement par concours, les affectations, la formation, la notation, les promotions etc. Ce modèle n'est pas spécifique des seuls militaires et la gestion collective des postes déborde parfois du seul champ des emplois publics de fonctionnaires. Donc il y a bien un modèle professionnel qui existe en France : c'est celui des corps. La proposition de Freyssinet consiste-t-elle à étendre largement le mode de gestion de l,emploi public ? Ne convient-il pas d'y regarder à deux fois tant les performances de cette forme de gestion ne

8 On peut se référer au commentaire de Julien Bonnecase publié dans le Recueil Dalloz de 1920

9 Saglio J. 2002, Le modèle militaire et la représentation des salariés dans le système français de relations professionnelles, In Bernier C., Jobert A., Rainbird $H_{\text {., }}$ Saglio J. 2002, Formation, relations professionnelles et syndicalísme à l'heure de la société-monde, L’harmattan, Presses de I,Université Laval, Québec, Paris, 169-184. 
sont pas totalement évidentes, notamment en matière de dualisme et de précarité comme en matière d'emplois des jeunes.

\section{Retour au local}

Je trouve très intéressant de voir que Jacques Freyssinet participe du mouvement actuel dintérêt pour les régulations locales de relations professionnelles. Comment faire ce ré-investissement du local ? Je naime pas trop utiliser pour ce faire le concept de "district industriel". Il est connoté de complémentarité productive et consiste à considérer que lensemble industriel est assimilable à une entreprise éclatée. Avec $\mathrm{M}-\mathrm{F}$ Raveyre ${ }^{10}$, nous avions suggéré " système industriel localisé " pour suggérer quil y avait trois éléments de régulation imbriqués dans cette régulation locale: les relations professionnelles au sens strict, mais aussi la gestion des technologies et la gestion des concurrences entre firmes. L'ensemble des acteurs, y compris des syndicats, jouaient de ces trois éléments. En approfondissant cette analyse, et notamment en essayant de minimiser son côté trop fonctionnaliste où les champs daction seraient quasi pré-définis on peut avancer l,idée que limportant dans ces systèmes est que les acteurs rentrent dans des "stratégies de conflit-coopération" totalement différents des " mécanismes contractuels "11. Dans de telles situations, il n'y a pas de contrat au sens classique du terme : il y a au contraire indétermination sur la contre-prestation attendue de la prestation. Il y a conflit du fait de cette indétermination. Mais les protagonistes sont d/accord sur un point à savoir que c'est entre eux que cela va se jouer. Et de ce fait, il y a une dynamique positive qui s'enclenche. C'est parce que les acteurs sont proches et liés - et opposés - de multiples façons que lindétermination de la contre-prestation peut rester forte : le service commercial rendu à un " collègue " peut être soldé en passant une nouvelle commande, en soutenant une campagne électorale ou en introduisant le partenaire

10 Raveyre M.F., Saglio J. 1984, Les Systèmes Industriels Localisés : éléments Pour une analyse sociologique des ensembles de P.M.E. industriels, Sociologie $\mathcal{D} u \mathcal{T}^{\prime}$ ravail $\mathbf{n}^{\circ} \mathbf{2}$, $157-175$.

11 Saglio J. 1991, Échange social et identité collective dans les systèmes industriels, Sociologie du $\mathcal{T}_{\text {ravail, }} \mathrm{n}^{\circ} \mathbf{4}$, 529-544. 
dans un nouveau cercle de connaissances... L'embauche peut suivre ou précéder l'acquisition des connaissances.

Réfléchir au niveau local, c'est réfléchir à ce qui peut faire que ce type de gestion advienne. Crest sortir de l,idée quill y aurait un domaine de problèmes spécifique du niveau local. C'est plutôt venir à l,idée que promouvoir les régulations locales, c'est drabord un moyen d,impliquer les acteurs, et de les impliquer parce quil y a indétermination. Les acteurs s'y engagent parce qu'ils savent quainsi ils vont rentrer dans des jeux de conflits et de coopérations où il pourront gagner quelque chose. Crest à cette condition d,indétermination que limplication au niveau local peut être bénéfique. 